\title{
Improved sparse error recovery approach for detecting QAM signals in overloaded massive MIMO systems
}

\author{
Yacine Meslem \\ Laboratoire Télécommunications, \\ École Militaire Polytechnique, \\ B.P 17, Bordj el Bahri (16111), Algeria \\ yacinemeslem@hotmail.fr
}

\author{
Abeldjalil Aïssa-El-Bey \\ IMT Atlantique, \\ UMR CNRS 6285 Lab-STICC, \\ Brest F-29238, France \\ abdeldjalil.aissaelbey@imt-atlantique.fr
}

\author{
Mustapha Djeddou \\ Laboratoire Télécommunications, \\ École Militaire Polytechnique, \\ B.P 17, Bordj el Bahri (16111), Algeria \\ djeddou.mustapha@gmail.com
}

\begin{abstract}
With a convenient concatenation of a convex relaxation-based detector and a simple greedy algorithm, we propose an improved Post Detection Sparse error Recovery (PDSR) approach for massive Multiple Input Multiple Output (m-MIMO) systems that, in particular, transmit QAM signals. The proposed PDSR approach can perform well in situations, where the classical one, either acts poorly or completely fails. We further propose an Alternating Direction Method of Multipliers (ADMM)-based solver for the convex detector, which is advantageous in maintaining an affordable complexity to the overall proposed detection scheme. Numerical experiments show the efficiency of our approach, especially when applied to overloaded m-MIMO systems.
\end{abstract}

Index Terms-Massive MIMO (m-MIMO), Signal detection, Convex optimization, ADMM, Greedy algorithms, Compressive sensing,

\section{INTRODUCTION}

One of the challenging signal processing tasks for massive Multiple Input Multiple Output (m-MIMO) systems is the detection of the transmitted data. This is because, the optimal Maximum Likelihood (ML) detector is computationally intractable. Therefore, several suboptimal detectors based on various approaches has been proposed in the literature. Among them, linear detectors such as Minimum Mean Square Error (MMSE), Sphere Decoder (SD)-based detectors, e.g. [1], the Layered Tabu Search Algorithm (LTSA) in [2], detectors based on the finite alphabet-sparse recovery approach [3] such as [4], and the simplicity-based detector [5], to just name few.

Another approach for detecting m-MIMO signals refers to as Post Detection Sparse error Recovery (PDSR) has been introduced in [6]. It improves the accuracy of a (linear) detector, as long as this detector induces an error that can be reasonably modeled as a sparse signal. In a nutshell, the PDSR approach is made up of two stages. In the first stage, a conventional linear detector, usually MMSE, is employed to obtain an initial estimate of the transmitted symbols. Then, in the second stage, a greedy algorithm, which is a Compressive Sensing (CS) [7] technique, is performed to correct the symbols that are missed by the first detector [6]. In that way, this two-stage detection approach can provide better Bit Error Rate (BER) performance over the single-stage (linear) one, without a significant increase of the computational cost [6].

In view of enhancing the performance of the PDSR approach, several techniques have been proposed [8]-[10]. In [8], a method named Sparsity-Boosted Iterative Linear (SBIL) is presented. It uses, in a first step, the Generalized Orthogonal Matching Pursuit (gOMP) [11] greedy algorithm to only locate the symbols that are falsely detected by MMSE. In the second step, the locations of those erroneous symbols are isolated to construct a reduced-size MIMO system, and the Zero Forcing (ZF) detector is applied for correction. The process is iteratively repeated until a stopping criterion is reached. The work in [9] can be viewed as an extension to SBIL. Its main idea is to concatenate several MMSE estimates of several transmitted vectors in the first stage, before applying a sparse retrieving technique. The objective is to get a good level of sparsity that can make the application of a greedy algorithm more efficient. In [10], the same idea of concatenation is adopted, while the Multipath Matching Pursuit (MMP) [12] is the chosen greedy algorithm, and SD is used to correct the erroneously detected symbols.

While the aforementioned techniques outperform the original PDSR approach (see for e.g. [9], [10]), their performance are expected to severally degrade when they are applied to an overloaded (underdetermined) m-MIMO system. The reason for such expected behavior is simply the use of a linear detector (MMSE) in their first stage. This is because, the induced error of a linear detector cannot be guaranteed to be sparse in those transmission scenarios. This makes the allover PDSR approach to perform poorly or even completely fail.

In order to extend the PDSR approach to deal with situations where the m-MIMO system is overloaded, and/or when it uses a higher-order signaling, we propose the use of a convex relaxation-based detector (non-linear) in the first stage. By doing so, we guarantee that the induced error, to be treated in the second stage, is reasonably sparse and thereby, it can be efficiently retrieved by a simple greedy algorithm. In addition, we suggest to solve the detection problem, in the first stage, via an Alternating Direction of Multipliers Method (ADMM) 
algorithm aiming at providing a comparable computational cost to the conventional PDSR approach.

The rest of this paper is structured as follows. We give our system model in section II. In section III, we formulate the detection problem as a convex one and present our ADMM based solution. In section IV, we suggest a simplified SBIL algorithm to further enhance the performance of the convexbased detector. Section $\mathrm{V}$ is devoted to simulation results, and finally, we conclude this paper in section VI.

Useful Definitions and Notation: We define the orthogonal projection of a vector $\mathbf{v}$ onto a convex closed set $\mathcal{D}$ as $\Pi_{\mathcal{D}}(\mathbf{v})=\underset{\mathbf{a r g m i n}}{\operatorname{ar}}\left(\frac{1}{2}\|\mathbf{x}-\mathbf{v}\|_{2}^{2}\right)$, and the indicator function as $\mathcal{I}_{\mathcal{D}}(\mathbf{v})=\left\{\begin{array}{ll}\mathbf{x} \in \mathcal{D} & \mathbf{v} \in \mathcal{D} \\ +\infty & \mathbf{v} \notin \mathcal{D}\end{array}\right.$. We reserve uppercase boldface letters for matrices and lowercase boldface letters for vectors. We denote the $d \times d$ identity matrix by $\mathbf{I}_{d}$. The sets of real and complex numbers are denoted by $\mathbb{R}$ and $\mathbb{C}$, respectively. We use $\Re\{\cdot\}$ and $\Im\{\cdot\}$ for the real and imaginary part of a complex-valued vector, respectively. The symbol $(\cdot)^{H}$ stands for the Hermitian of a matrix (resp. vector). The comparison $\min (a, \mathbf{v})$, (resp. $\max (a, \mathbf{v}))$ where $a$ is scalar, is done element-wise. $\jmath=\sqrt{-1}$.

\section{SYSTEM MODEL}

We consider a spatially multiplexed m-MIMO system with $N_{t}$ antennas transmitting $N_{t}$ different symbols, and $N_{r}$ antennas for reception. The m-MIMO system is called overloaded or underdetermined when, $N_{r}<N_{t}$. The channel response is denoted by $\mathbf{H} \in \mathbb{C}^{N_{r} \times N_{t}}$, and their elements are i.i.d random variables distributed according to the standard complex Gaussian distribution. The received signal $\mathbf{y}$ is given by

$$
\mathbf{y}=\mathbf{H x}+\boldsymbol{\nu},
$$

where, $\mathbf{x} \in \mathcal{Q}^{N_{t}}$ and $\mathcal{Q}$ is the modulation set (alphabet). The random vector $\boldsymbol{\nu} \in \mathbb{C}^{N_{t}}$ follows a multivariate complex Gaussian distribution with zero mean and $\sigma^{2} \mathbf{I}_{N_{r}}$ covariance matrix, and it represents the receiver noise.

The ML detection problem reads

$$
\begin{array}{ll}
\text { minimize } & \frac{1}{2}\|\mathbf{y}-\mathbf{H x}\|_{2}^{2} \\
\text { subject to } & \mathbf{x} \in \mathcal{Q}^{N_{t}} .
\end{array}
$$

Throughout this paper, we focus the m-MIMO detection problem for $L$-QAM square alphabets, for which the ML detection problem (2) can be equivalently written as

$$
\begin{array}{ll}
\text { minimize } & \frac{1}{2}\|\mathbf{y}-\mathbf{H x}\|_{2}^{2} \\
\text { subject to } & \Re\{\mathbf{x}\} \in \mathcal{A}^{N_{t}} \\
& \Im\{\mathbf{x}\} \in \mathcal{A}^{N_{t}} .
\end{array}
$$

The set $\mathcal{A}=\{-a,-a+2, \ldots, a-2, a\}$, where $a=\sqrt{L}-1$.

\section{First Stage Detector: Convex Relaxation and ADMM SOLUTION}

From CS perspective, the first stage detector, in PDSR, plays the role of a sparse transform. It converts the dense vector $\mathbf{x}$ into a sparse one, which is the difference between $\mathbf{x}$ and its estimate [6]. In light of the foregoing, linear detectors

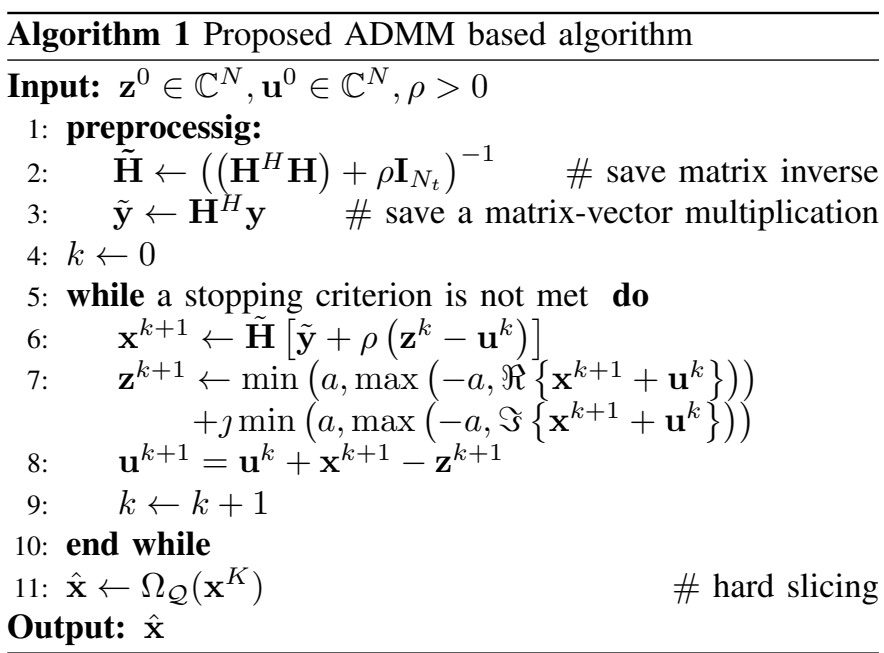

cannot be, in general, considered as good sparse transforms and therefore, a more reliable detector is required.

The convex relaxation trick is commonly used when dealing with hard optimization problems like (3). The idea is to solve a convex minimization problem that approximates the original hard one. Usually, this technique results in a good approximate solution. By exploring this trick, we can formulate the detection problem, in the first stage, as

$$
\begin{array}{ll}
\operatorname{minimize} & \frac{1}{2}\|\mathbf{y}-\mathbf{H} \mathbf{x}\|_{2}^{2} \\
\text { subject to } & \|\Re\{\mathbf{x}\}\|_{\infty} \leq a \\
& \|\Im\{\mathbf{x}\}\|_{\infty} \leq a .
\end{array}
$$

Here, we have relaxed the discrete set $\mathcal{A}^{N_{t}}$ into its convex hull (the smallest convex set that contains $\mathcal{A}^{N_{t}}$ ), which is the infinity ball $\mathcal{B}_{\infty}(0, a)$ of center 0 and radius $a$, defined as $\mathcal{B}_{\infty}(0, a)=\left\{\mathbf{x} \in \mathbb{R}^{n} \mid\|\mathbf{x}\|_{\infty} \leq a\right\}$. It worth mentioning that, while it is relatively simple to understand the intuition why $\mathcal{B}_{\infty}(0, a)$ is the convex hull of $\mathcal{A}^{N_{t}}$, a rigorous proof, however, is not that simple.

For the solution of (4), we rely on the ADMM framework. This choice is motivated by the fact that applying ADMM to well-structured convex problems leads to simple and lowcomplexity algorithms [13]. We derive hereafter, our ADMMbased solution to (4).

Problem (4), can be put in a split form, as follows

$$
\operatorname{minimize} \quad \frac{1}{2}\|\mathbf{y}-\mathbf{H x}\|_{2}^{2}+\mathcal{I}_{\mathcal{B}_{\infty}}(\Re\{\mathbf{x}\})+\mathcal{I}_{\mathcal{B}_{\infty}}(\Im\{\mathbf{x}\})
$$$$
\text { subject to } \mathbf{x}=\mathbf{z} \text {, }
$$

where, $\mathbf{z} \in \mathbb{C}^{N_{t}}$ is a dummy variable. Note that for lightening the notation, we refer to $\mathcal{B}_{\infty}(0, a)$ hence-after, as simply $\mathcal{B}_{\infty}$. The scaled-form of the augmented Lagrangian associated with (5) is given by

$$
\begin{aligned}
\mathcal{L}_{\rho}(\mathbf{x}, \mathbf{z}, \mathbf{u}) & =\frac{1}{2}\|\mathbf{y}-\mathbf{H} \mathbf{x}\|_{2}^{2}+\mathcal{I}_{\mathcal{B}_{\infty}}(\Re\{\mathbf{x}\})+\mathcal{I}_{\mathcal{B}_{\infty}}(\Im\{\mathbf{x}\}) \\
& +\frac{\rho}{2}\|\mathbf{x}-\mathbf{z}+\mathbf{u}\|_{2}^{2},
\end{aligned}
$$

where, $\rho>0$ is a parameter. ADMM proceeds in three steps. An alternate minimization of the augmented Lagrangian (6) 


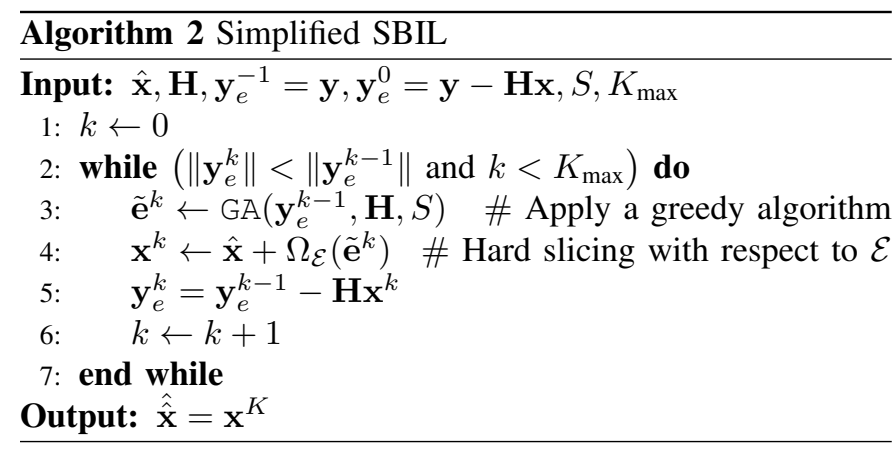

with respect to the primal variables; first $\mathbf{x}$, then $\mathbf{z}$, and finally an update of the dual variable $\mathbf{u}$.

a) $x$-update: At iteration $(k+1), \mathbf{x}^{k+1}$ is the minimizer of $\mathcal{L}_{\rho}\left(\mathbf{x}, \mathbf{z}^{k}, \mathbf{u}^{k}\right)$. Thus, to obtain $\mathbf{x}^{k+1}$, we solve

$$
\text { minimize } \frac{1}{2}\|\mathbf{y}-\mathbf{H x}\|_{2}^{2}+\frac{\rho}{2}\left\|\mathbf{x}-\mathbf{z}^{k}+\mathbf{u}^{k}\right\|_{2}^{2} .
$$

The solution of (7) can be easily obtained using Wintiger calculus [14] for differentiable functions on $\mathbb{C}^{N_{t}}$, and hence,

$$
\mathbf{x}^{k+1}=\left(\mathbf{H}^{H} \mathbf{H}+\rho \mathbf{I}_{N_{t}}\right)^{-1}\left(\mathbf{H}^{H} \mathbf{y}+\rho\left[\mathbf{z}^{k}-\mathbf{u}^{k}\right]\right) .
$$

b) z-update: This step reduces to solve

$$
\begin{aligned}
\operatorname{minimize} & \mathcal{I}_{\mathcal{B}_{\infty}}(\Re\{\mathbf{z}\})+\mathcal{I}_{\mathcal{B}_{\infty}}(\Im\{\mathbf{z}\}) \\
& +\frac{\rho}{2}\left\|\mathbf{x}^{k+1}+\mathbf{u}^{k}-\mathbf{z}\right\|_{2}^{2} .
\end{aligned}
$$

Observe that, problem (9) is separable in $\Re\{\mathbf{z}\}$ and $\Im\{\mathbf{z}\}$. Thereby, we only need to determine the projection of a real vector onto $\mathcal{B}_{\infty}$. This is given by the lemma below.

Lemma 1. The projection $\Pi_{\mathcal{B}_{\infty}}(\mathbf{v})$ of a vector $\mathbf{v} \in \mathbb{R}^{n}$ onto the set $\mathcal{B}_{\infty}$ is given by

$$
\Pi_{\mathcal{B}_{\infty}}(\mathbf{v})=\min (a, \max (-a, \mathbf{v})) .
$$

Proof. Here is one simple way to prove Lemma 1 . The first thing to notice is that projection problem can be equivalently written as $n$ separable optimization problems as follows

$$
\begin{array}{ll}
\operatorname{minimize} & \left(v_{i}-w_{i}\right)^{2} \\
\text { subject to } & \left|w_{i}\right| \leq a, \forall i=1, \ldots, n
\end{array}
$$

We distinguish two cases. The first one is when $\left|v_{i}\right| \leq a$. In this case, $v_{i}$ is already in the set $[-a, a]$ and hence, its projection is equal to itself, i.e., $v_{i}$. The remaining case is when $\left|v_{i}\right|>a$. Here, the projection of $v_{i}$ is $a$ when $v_{i}>a$ and $-a$ when $v_{i}<-a$. This is so done to maximally diminishing the objective function. By compactly writing those solutions, we obtain the above formula.

The proposed ADMM-based algorithm for solving (4) is detailed in Algorithm 1. It worth mentioning that its computational complexity is dominated by the matrix inversion, which is required only once. The rest of operations require at most $\mathcal{O}\left(N_{t}^{2}\right)$. Therefore, the estimated complexity of Algorithm 1 is of the order $\mathcal{O}\left(N_{t}^{3}\right)$, which comparable to the computational cost of linear detectors. Especially, if we know that the average number of iterations required for Algorithm 1 to converge is about 40 in all the conducted simulations (see, Section V).

\section{Second Stage: Sparse Error Recovery}

In PDSR approach, the second stage is concerned with the recovery of a sparse signal, which is the error $\mathbf{e} \in \mathcal{E}^{N_{t}}$ induced by the first stage detector. Mathematically,

$$
\mathbf{e}=\mathbf{x}-\hat{\mathbf{x}}
$$

where, $\hat{\mathbf{x}}$ is the output of the first stage detector. The set $\mathcal{E}$ is called the error alphabet. By simple algebraic manipulations, we get the reception model for $\mathbf{e}$ as

$$
\mathbf{y}_{e}=\mathbf{H e}+\boldsymbol{\nu},
$$

where, $\mathbf{y}_{e}=\mathbf{y}-\mathbf{H} \hat{\mathbf{x}}$. Using (11), the goal now, is to recover e in order to improve the performance of the convex-based detector (Algorithm 1). Assuming that the first stage detector is reliable-enough, e can be fairly considered sparse. Thus, for an efficient recovery of e, one can resort on CS techniques in order to solve the following sparse-recovery problem

$$
\begin{array}{ll}
\text { minimize } & \left\|\mathbf{y}_{e}-\mathbf{H e}\right\|_{2}^{2} \\
\text { subject to } & \|\mathbf{e}\|_{0} \leq S,
\end{array}
$$

where $S$ is un upper-bound on the sparsity level of e.

Problems like (12) are, now, well-understood, and they are efficiently solvable via various approaches. For a recent review on these approaches, the interested reader may refer to [15].

Greedy algorithms are popular in solving sparse recovery problems. They are characterized by their simplicity together with their ability, under some conditions, to provide solutions at the same order of accuracy as those obtained with other approaches namely, convex-approximation [16]. Further, on the side of computational complexity, they are attractive.

Based on the greedy approach, we propose an algorithm, which we call simplified SBIL (Algorithm 2), to recover e. We use a greedy algorithm such as gOMP [11] or the Compressive Sampling Matching Pursuit (CoSaMP) [17], in an iterative manner, as the SBIL method [8] does. We omit, however, the ZF step and directly perform a hard slicing in order to simplify and speed up the process. The stopping criteria are the same as in SBIL, and we heuristically set $S=\left\lceil\frac{N_{t}}{10}\right\rceil$.

\section{NUMERICAL EXPERIMENTS}

In this section, the BER performance of the proposed PDSR approach is demonstrated via numerical simulation. The experiments are done for two QAM alphabets; a lower-order 4-QAM and a higher-order 16-QAM, and different system dimensions $\left(N_{t} \times N_{r}\right)$, where the focus is on the overloaded case. The Rayleigh flat fading channel is considered. For comparison purpose, we include MMSE ("MMSE"), the SBIL method as it is introduced in [8] ("SBIL") and the Iterative Weighted Sum Of Absolute Values [18] ("IW-SOAV"), which is a convex-optimization-based detection scheme for overloaded m-MIMO systems, as references. The parameters of IW-SOAV for both regularization and for the RachfordDouglas algorithm are set as stated in [18]. Also, we show the BER curves of the proposed detector in the first stage ("proposed (first stage only)") to get an insight on the gain when Algorithm 1 is concatenated with the simplified SBIL. 


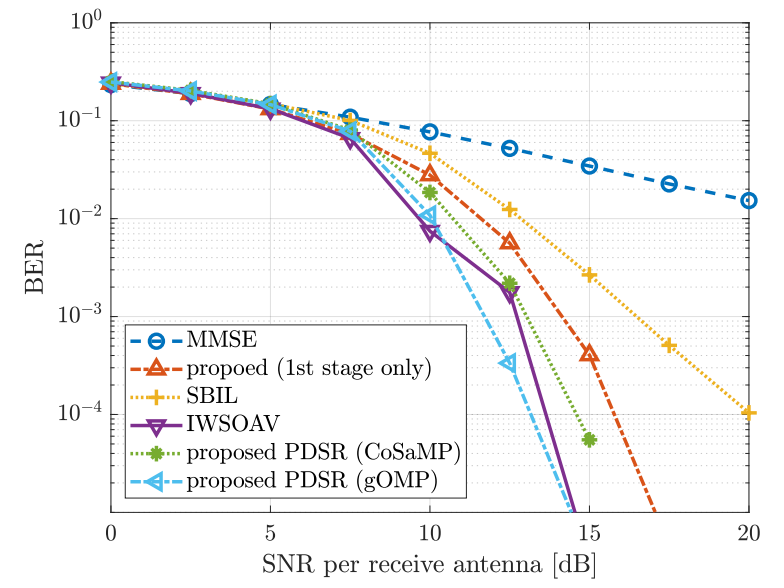

Fig. 1. BER performance of $128 \times 112$ MIMO system with 4-QAM

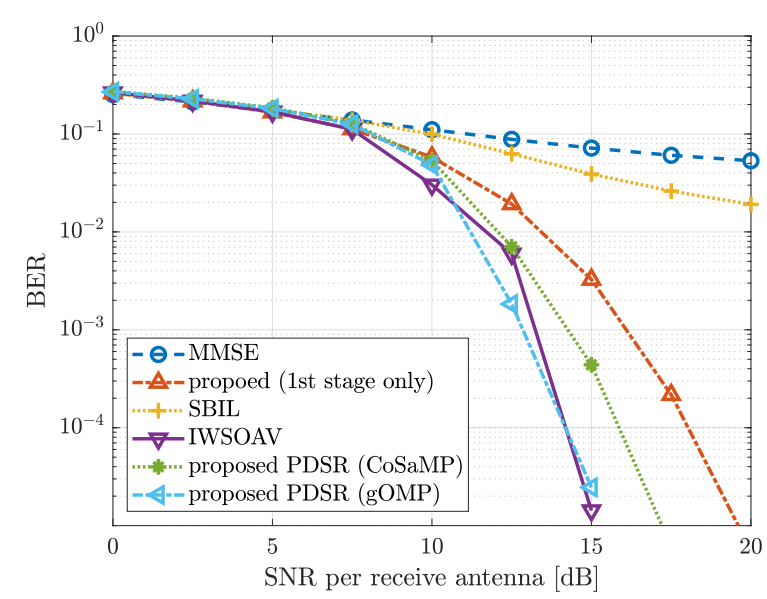

Fig. 2. BER performance of $128 \times 96$ MIMO system with $4-$ QAM

Figs. 1 and 2 show the BER curves of two overloaded mMIMO systems $128 \times 112$ and $128 \times 96$, respectively, both transmit 4-QAM symbols. As expected, the conventional PDSR (SBIL) performs poorly. Further, it is even far from the performance of Algorithm 1 alone. As for the proposed PDSR, it performs as well as the IW-SOAV method when the gOMP is used. We should, however, mention that the curves for IW-SOAV are obtained for 5 iterations. At each, a convex optimization problem of the same order of complexity as Algorithm 1, is solved, while the simplified SBIL runs at most for 2 iterations. The gap of performance when, CoSaMP is used, confirms the superiority of gOMP than other greedy algorithms, as it can achieve better recovery probability for signals of low-sparsity level [11]. Note that, the gain of performance when the allover PDSR approach is employed rather than the convex detector alone, is more than $4 \mathrm{~dB}$ in the more overloaded case $(128 \times 96)$. This is due to the convenient choice of the proposed convex detector as a sparse transform.

In, Fig. 3 and Fig. 4, we show the BER performance of two m-MIMO systems using 16-QAM alphabet. The first is a determined $96 \times 96$ system and the second is an overload $96 \times 90$ one. IW-SOAV is no more included in the comparison,

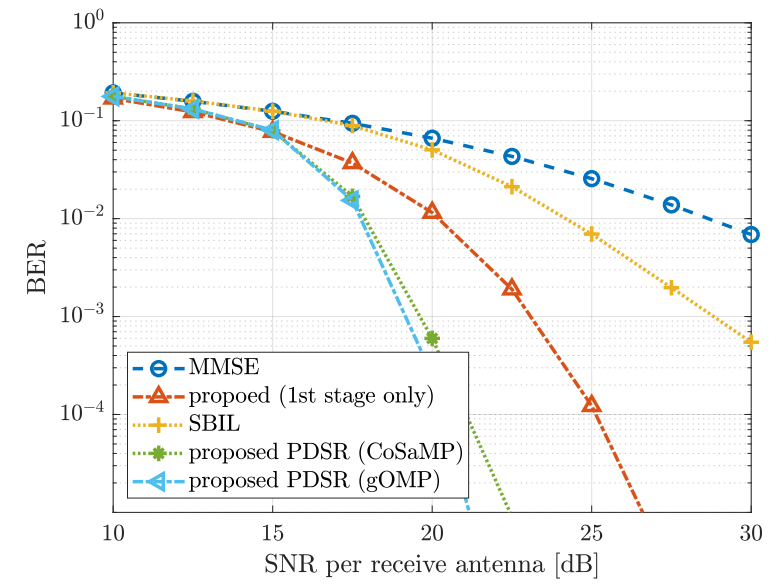

Fig. 3. BER performance of $96 \times 96$ MIMO system with $16-$ QAM

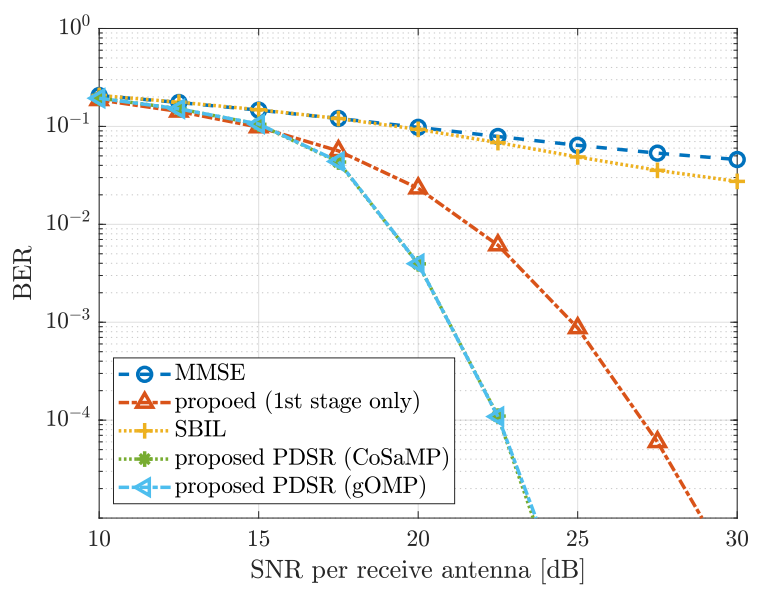

Fig. 4. BER performance of $96 \times 90$ MIMO system with $16-$ QAM

since its parameters for this signaling scheme are not available. Once more, the proposed approach performs well in both cases. It improves about $4 \mathrm{~dB}$ on Algorithm 1 , while the classical PDSR completely fails. Again, this failure is because of the use of a linear detector as a sparse transform. Note that the gap between gOMP and CoSaMP reduces and they performs the same in the overloaded case. This may be explained by the fact that the sparsity-level of the error, that the convex detector provides, for those transmission scenarios, is in the range where CoSaMP and gOMP have the same performance [11].

\section{CONCLUSION}

We have proposed an improved PDSR approach for detecting QAM signals in m-MIMO systems. The proposed approach relies on a convex detector, that, on one hand, can efficiently transform dense QAM signals into sparse error vectors, even in overloaded transmission scenarios. And on the other hand, can be solved with a low-complexity algorithm, thanks to ADMM. The conducted simulations demonstrate that the proposed approach can be competitive to existing detection methods for m-MIMO signals, namely in the overloaded case. 


\section{REFERENCES}

[1] C. Zheng, X. Chu, J. McAllister, and R. Woods, "Real-valued fixedcomplexity sphere decoder for high dimensional QAM-MIMO systems," IEEE Transactions on Signal Processing, vol. 59, no. 9, pp. 4493-4499, Sep. 2011

[2] N. Srinidhi, T. Datta, A. Chockalingam, and B. S. Rajan, "Layered tabu search algorithm for large-MIMO detection and a lower bound on ML performance," IEEE Transactions on Communications, vol. 59, no. 11, pp. 2955-2963, November 2011.

[3] A. Aïssa-El-Bey, D. Pastor, S. M. A. Sbaï, and Y. Fadlallah, "Sparsitybased recovery of finite alphabet solutions to underdetermined linear systems," IEEE Transactions on Information Theory, vol. 61, no. 4, pp. 2008-2018, 2015.

[4] Y. Fadlallah, A. Aïssa-El-Bey, K. Amis, D. Pastor, and R. Pyndiah, "New iterative detector of MIMO transmission using sparse decomposition," IEEE Transactions on Vehicular Technology, vol. 64, no. 8, pp. 34583464, Aug 2015.

[5] Z. Hajji, A. Aïssa-El-Bey, and K. Amis, "Simplicity-based recovery of finite-alphabet signals for large-scale MIMO systems," Digital Signal Processing, vol. 80, pp. $70-82,2018$.

[6] J. W. Choi and B. Shim, "New approach for massive MIMO detection using sparse error recovery," in 2014 IEEE Global Communications Conference, Dec 2014, pp. 3754-3759.

[7] D. L. Donoho, "Compressed sensing," IEEE Transactions on Information Theory, vol. 52, no. 4, pp. 1289-1306, April 2006.

[8] X. Peng, W. Wu, J. Sun, and Y. Liu, "Sparsity-boosted detection for large MIMO systems," IEEE Communications Letters, vol. 19, no. 2, pp. 191-194, Feb 2015.

[9] Abhishek, A. K. Sah, and A. K. Chaturvedi, "Improved sparsity behaviour and error localization in detectors for large MIMO systems," in 2016 IEEE Globecom Workshops (GC Wkshps), 2016, pp. 1-6.

[10] A. K. Sah and A. K. Chaturvedi, "An MMP-based approach for detection in large MIMO systems using sphere decoding," IEEE Wireless Communications Letters, vol. 6, no. 2, pp. 158-161, April 2017.

[11] J. Wang, S. Kwon, and B. Shim, "Generalized orthogonal matching pursuit," IEEE Transactions on Signal Processing, vol. 60, no. 12, pp. 6202-6216, Dec 2012.

[12] S. Kwon, J. Wang, and B. Shim, "Multipath matching pursuit," IEEE Transactions on Information Theory, vol. 60, no. 5, pp. 2986-3001, May 2014.

[13] S. Boyd, N. Parikh, E. Chu, B. Peleato, J. Eckstein et al., "Distributed optimization and statistical learning via the alternating direction method of multipliers," Foundations and Trends $®$ in Machine learning, vol. 3, no. 1, pp. 1-122, 2011.

[14] D. H. Brandwood, "A complex gradient operator and its application in adaptive array theory," IEE Proceedings F - Communications, Radar and Signal Processing, vol. 130, no. 1, pp. 11-16, February 1983.

[15] E. Crespo Marques, N. Maciel, L. Naviner, H. Cai, and J. Yang, "A review of sparse recovery algorithms," IEEE Access, vol. 7, pp. 1300$1322,2019$.

[16] J. A. Tropp, "Greed is good: algorithmic results for sparse approximation," IEEE Transactions on Information Theory, vol. 50, no. 10, pp. 2231-2242, Oct 2004.

[17] D. Needell and J. Tropp, "CoSaMP: Iterative signal recovery from incomplete and inaccurate samples," Applied and Computational Harmonic Analysis, vol. 26, no. 3, pp. 301 - 321, 2009.

[18] R. Hayakawa and K. Hayashi, "Convex optimization-based signal detection for massive overloaded MIMO systems," IEEE Transactions on Wireless Communications, vol. 16, no. 11, pp. 7080-7091, Nov 2017. 